\title{
Evaluation of the Zeiss retinal vessel analyser
}

Kaija Polak, Guido Dorner, Barbara Kiss, Elzbieta Polska, Oliver Findl, Georg Rainer, Hans-Georg Eichler, Leopold Schmetterer

\begin{abstract}
Aim-To investigate the reproducibility and sensitivity of the Zeiss retinal vessel analyser, a new method for the online determination of retinal vessel diameters in healthy subjects.

Methods-Two model drugs were administered, a peripheral vasoconstrictor (the $\alpha$ receptor agonist phenylephrine) and a peripheral vasodilator (the nitric oxide donor sodium nitroprusside) in stepwise increasing doses. Nine healthy young subjects were studied in a placebo controlled double masked three way crossover design. Subjects received intravenous infusions of either placebo or stepwise increasing doses of phenylephrine $(0.5,1$, or $2 \mu \mathrm{g} / \mathrm{kg} / \mathrm{min}$ ) or sodium nitroprusside $(0.5,1$, or $2 \mu \mathrm{g} / \mathrm{kg} / \mathrm{min})$. Retinal vessel diameters were measured with the new Zeiss retinal vessel analyser. Retinal leucocyte velocity, flow, and density were measured with the blue field entoptic technique. The reproducibility of measurements was assessed with coefficients of variation and intraclass correlation coefficients.
\end{abstract}

Results-Placebo and phenylephrine did not influence retinal haemodynamics, although the $\alpha$ receptor antagonist significantly increased blood pressure. Sodium nitroprusside induced a significant increase in retinal venous and arterial diameters $(p<0.001$ each), leucocyte density $(p=0.001)$, and leucocyte flow $(p=0.024)$ despite lowering blood pressure to a significant degree. For venous and arterial vessel size measurements short term coefficients of variation were $1.3 \%$ and $2.6 \%$ and intraclass correlation coefficients were 0.98 and 0.96 , respectively. The sensitivity was between $3 \%$ and $5 \%$ for retinal veins and $5 \%$ and $7 \%$ for retinal arteries.

Conclusions-These data indicate that the Zeiss retinal vessel analyser is an accurate system for the assessment of retinal diameters in healthy subjects. In addition, nitric oxide appears to have a strong influence on retinal vascular tone.

(Br f Ophthalmol 2000;84:1285-1290)

There is evidence that a variety of eye diseases are associated with alterations in retinal blood flow. Hence, there is considerable interest in studying retinal blood flow in humans. One method to gain insight into retinal haemodynamics is to determine the diameter of retinal vessels. A variety of methods have been proposed to measure retinal vessel size in vivo. $^{1-6}$

Obviously the optimal technique for the assessment of retinal blood flow should provide a high reproducibility and sensitivity. In addition, an adequate time resolution is required if pharmacological or physiological interventions are to be studied. Recently, a commercially available system for the real time assessment of retinal vessel diameters in vivo has been introduced (Zeiss Retinal Vessel Analyzer, Oberkochen, Germany). The aim of the present study was to evaluate this system in healthy young subjects. For this purpose we administered two model drugs, a peripheral vasoconstrictor (the $\alpha$ adrenoceptor agonist phenylephrine) and a peripheral vasodilator (the nitric oxide donor sodium nitroprusside) in a randomised, placebo controlled study. The results obtained with this technique were compared with the results measured with the blue field entoptic technique, which assesses leucocyte movement in perifoveal retinal capillaries.

\section{Methods}

SUBJECTS

The study protocol was approved by the ethics committee of Vienna University School of Medicine and followed the guidelines of the Declaration of Helsinki. Nine healthy male (age range 20-27 years, mean 23.6 (SD 2.0)) non-smoking volunteers participated in this study after signing written informed consent. Each subject passed a screening examination that included medical history and physical examination, 12 lead electrocardiogram, complete blood count, activated partial thromboplastin time, thrombin time, fibrinogen, clinical chemistry (sodium, potassium, creatinine, uric acid, glucose, cholesterol, triglycerides, alanine aminotransferase, aspartate aminotransferase, $\gamma$ glutamyltransferase, alkaline phosphatase, total bilirubin, total protein), hepatitis A, B, C, and HIV serology, urine analysis, and a urine drug screen. Subjects were excluded if any abnormality was found as part of the screening unless the investigators considered an abnormality as clinically irrelevant. An ophthalmic examination was performed in each subject before the study day. Inclusion criteria were normal ophthalmic findings, ametropia of less than 3 dioptres, and anisometropia of less than 1 dioptre. In all subjects the right eye was studied.

\section{EXPERIMENTAL DESIGN}

The study was performed in a randomised, placebo controlled, balanced three way crossover design. Three study days with washout
Accepted for publication 16 May 2000 
Table 1 Coefficient of variation (CV) and intraclass correlation coefficients ( $\kappa$ ) for measurements of venous and arterial diameters with the Zeiss retinal vessel analyser and measurements of retinal leucocyte flow with the blue field entoptic technique. Data are presented for short term and day to day variability

\begin{tabular}{|c|c|c|c|c|}
\hline & \multicolumn{2}{|c|}{ Short term variability } & \multicolumn{2}{|c|}{ Day to day variability } \\
\hline & $C V$ & $\kappa$ & $C V$ & $\kappa$ \\
\hline Venous diameter & $1.3 \%$ & 0.98 & $4.4 \%$ & 0.90 \\
\hline Arterial diameter & $2.6 \%$ & 0.96 & $5.2 \%$ & 0.87 \\
\hline Retinal blood flow & $11.4 \%$ & 0.83 & $17.6 \%$ & 0.64 \\
\hline
\end{tabular}

Table 2 Baseline ocular haemodynamic variables at the three study days. Data are presented as means (SEM) $(n=9)$

\begin{tabular}{llll}
\hline & Day 1 & Day 2 & Day 3 \\
\hline Venous diameter (arbitrary units) & $159(7)$ & $157(6)$ & $155(8)$ \\
Arterial diameter (arbitrary units) & $139(10)$ & $132(8)$ & $137(9)$ \\
Leucocyte velocity (arbitrary units) & $1.10(0.09)$ & $1.09(0.08)$ & $1.03(0.08)$ \\
Leucocyte density (arbitrary units) & $103(17)$ & $103(18)$ & $113(17)$ \\
Leucocyte flow (arbitrary units) & $117(19)$ & $114(23)$ & $125(21)$ \\
\hline
\end{tabular}

periods of at least two study days were scheduled for each subject. On the 3 days the subjects received intravenous infusions of either phenylephrine (Neosynephrine, Winthrop Breon Laboratories, NY, USA; dose $0.5,1,2 \mu \mathrm{g} / \mathrm{kg} / \mathrm{min}$ ), or sodium nitroprusside (Nipruss-Trockensubstanz zur Infusionsbereitung, Sanol-Schwarz, Monheim, Germany; dose $0.5,1,2 \mu \mathrm{g} / \mathrm{kg} / \mathrm{min}$ ), or physiological saline solution as placebo. In order to obtain baseline values a 12 minute physiological saline infusion period was scheduled at each study day before the active drug or placebo was administered. The duration of each infusion step was also 12 minutes. The doses of these drugs were based on the results of a previous trial. $^{7}$

All subjects were studied after dilating the pupil with one drop of tropicamide (Mydriatikum Agepha, Agepha, Vienna, Austria). After a resting period in a sitting position, which lasted at least 20 minutes, the infusions were started. Baseline measurements with the Zeiss retinal vessel analyser and the blue field entoptic technique were performed during the last 5 minutes of the first infusion step. Measurements were then repeated during the last 5 minutes of each infusion step. Blood pressure was measured in 5 minute intervals during the study period. Pulse rate and real time ECG were monitored continuously.

ZEISS RETINAL VESSEL ANALYSER (RVA)

The Zeiss RVA is a commercially available system which comprises a fundus camera (Zeiss FF 450, Jena, Germany), a video camera, a real time monitor, and a personal computer with an analysing software for the accurate determination of retinal arterial and venous diameters. ${ }^{8}$ Retinal vessel diameters are analysed in real time with a maximum frequency of $50 \mathrm{~Hz}$. This means that a maximum of 25 vessel diameter readings can be obtained per second. For this purpose the fundus is imaged onto the charge coupled device chip of the video camera. The consecutive fundus images are digitised using a frame grabber. In addition, the fundus image can be inspected on the real time monitor and, if necessary, stored on a video recorder. Evaluation of the retinal vessel diameters can either be done on line or off line from the recorded video tapes.

Because of the absorbing properties of haemoglobin each blood vessel has a specific transmittance profile. Measurement of retinal vessel diameters is based on adaptive algorithms using these specific profiles. Whenever a specific vessel profile is recognised the RVA is able to follow this vessel as long as it appears within the measurement window. This means that the system is able to automatically correct for alterations in luminance as induced, for instance, by slight eye movements. If the requirements for the assessment of retinal vessel diameters are not being fulfilled, as occurs during blinks, the system automatically stops the measurement of vessel diameter. As soon as an adequate fundus image is achieved again, measurement of vessel diameters restarts automatically.

To select a region of interest the user defines a rectangle on the screen of the real time monitor. This window can either include a retinal artery or a retinal vein or both. Thereafter the measurement of vessel diameters can be started. Retinal vessel diameter is then calculated along the arterial or venous segment, which lies within the rectangle. If the region of interest consists of an artery and a vein, both vessel diameters are recorded simultaneously. As long as the vessels under study are within the selected rectangle the system automatically corrects for eye movements. This is made possible by the adaptive nature of the diameter analysis. Hence vessel diameter can be recorded as a function of time as well as a function of the position along the vessel. In contrast with other procedures, which have been proposed for the determination of retinal vessel size, this system measures diameters only in relative units.

BLUE FIELD ENTOPTIC TECHNIQUE

This non-invasive method is described in detail by Riva and Petrig. ${ }^{9}$ The blue field entoptic phenomenon can best be seen by looking into a blue light with a narrow optical spectrum at a wavelength of approximately $430 \mathrm{~nm}$. Under these conditions corpuscles can be seen moving in an area of 10-15 degrees of arc radius centred at the fovea. Most probably this phenomenon is caused by the fact that red, but not white, blood cells absorb short wavelength light. Thus the passage of a white blood cell is perceived as a flying corpuscle. For determination of retinal haemodynamic parameters a stimulated particle field is shown to the subjects under study. By comparison with their own entoptic observation subjects can adjust the number of white blood cells and the mean flow velocity from which the retinal leucocyte flow can be calculated. In the present studies at least five matching trials were performed by each subject. Only values with a coefficient of variation of less then $15 \%$ were considered accurate. Subjects who did not reach the required reproducibility were excluded from the study. 
Table 3 Effects of placebo (physiological saline solution) or stepwise increasing doses of sodium nitroprusside, or phenylephrine on systolic and diastolic blood pressure and pulse rate. Data are presented as means (SEM) $(n=9)$

\begin{tabular}{llll}
\hline & $\begin{array}{l}\text { Systolic blood pressure } \\
(\mathrm{mm} \text { Hg) }\end{array}$ & $\begin{array}{l}\text { Diastolic blood pressure } \\
(\mathrm{mm} \mathrm{Hg})\end{array}$ & Pulse rate (beats/min) \\
\hline $\begin{array}{lll}\text { Placebo day } \\
\text { Baseline }\end{array}$ & $114.5(9.2)$ & $60.9(7.4)$ & $70.7(10.3)$ \\
Syringe 1 & $113.4(7.6)$ & $61.1(5.4)$ & $68.6(11.6)$ \\
Syringe 2 & $112.9(3.3)$ & $60.6(6.5)$ & $67.3(8.8)$ \\
Syringe 3 & $109.6(11.1)$ & $59.9(6.9)$ & $67.2(10.6)$ \\
Phenylephrine & & & \\
Baseline & $113.8(8.5)$ & $57.8(9.2)$ & $61.2(5.9)$ \\
$0.5 \mu \mathrm{g} / \mathrm{kg} / \mathrm{min}$ & $121.0(9.2)$ & $62.5(10.9)$ & $59.2(9.5)$ \\
$1.0 \mu \mathrm{g} / \mathrm{kg} / \mathrm{min}$ & $132.5(11.0)$ & $69.1(10.4)$ & $48.9(8.3)$ \\
$2.0 \mu \mathrm{g} / \mathrm{kg} / \mathrm{min}$ & $145.4(12.6)$ & $81.5(11.1)$ & $45.6(7.6)$ \\
Sodium nitroprusside & & & \\
$\mathrm{Baseline}$ & $113.7(8.4)$ & $61.2(5.9)$ & $70.8(6.1)$ \\
$0.5 \mu \mathrm{g} / \mathrm{kg} / \mathrm{min}$ & $101.5(11.9)$ & $59.2(9.5)$ & $81.6(6.8)$ \\
$1.0 \mu \mathrm{g} / \mathrm{kg} / \mathrm{min}$ & $98.9(9.7)$ & $48.9(8.3)$ & $83.0(6.7)$ \\
$2.0 \mu \mathrm{g} / \mathrm{kg} / \mathrm{min}$ & $96.6(6.5)$ & $45.6(7.6)$ & \\
\hline
\end{tabular}

SYSTEMIC HAEMODYNAMICS

Systolic, diastolic, and mean blood pressures (SBP, DBP, MAP) were measured on the upper arm by an automated oscillometric device (HP-CMS patient monitor, Hewlett Packard, Palo Alto, CA, USA). Pulse rate (PR) was automatically recorded from a finger pulse oxymetric device (HP-CMS patient monitor).

DATA ANALYSIS

To quantify the short term variability and the day to day variability of the measurements intraclass correlation coefficients $(\kappa)$ for retinal venous and arterial diameters measured with the Zeiss retinal vessel analyser , as well as for retinal leucocyte flow measured with the blue field entoptic technique, were calculated. The calculation of $\kappa$ is based on a repeated measure ANOVA model using the variance among subjects (vs), the variance among measurements (vm), and the residual error variance (ve):
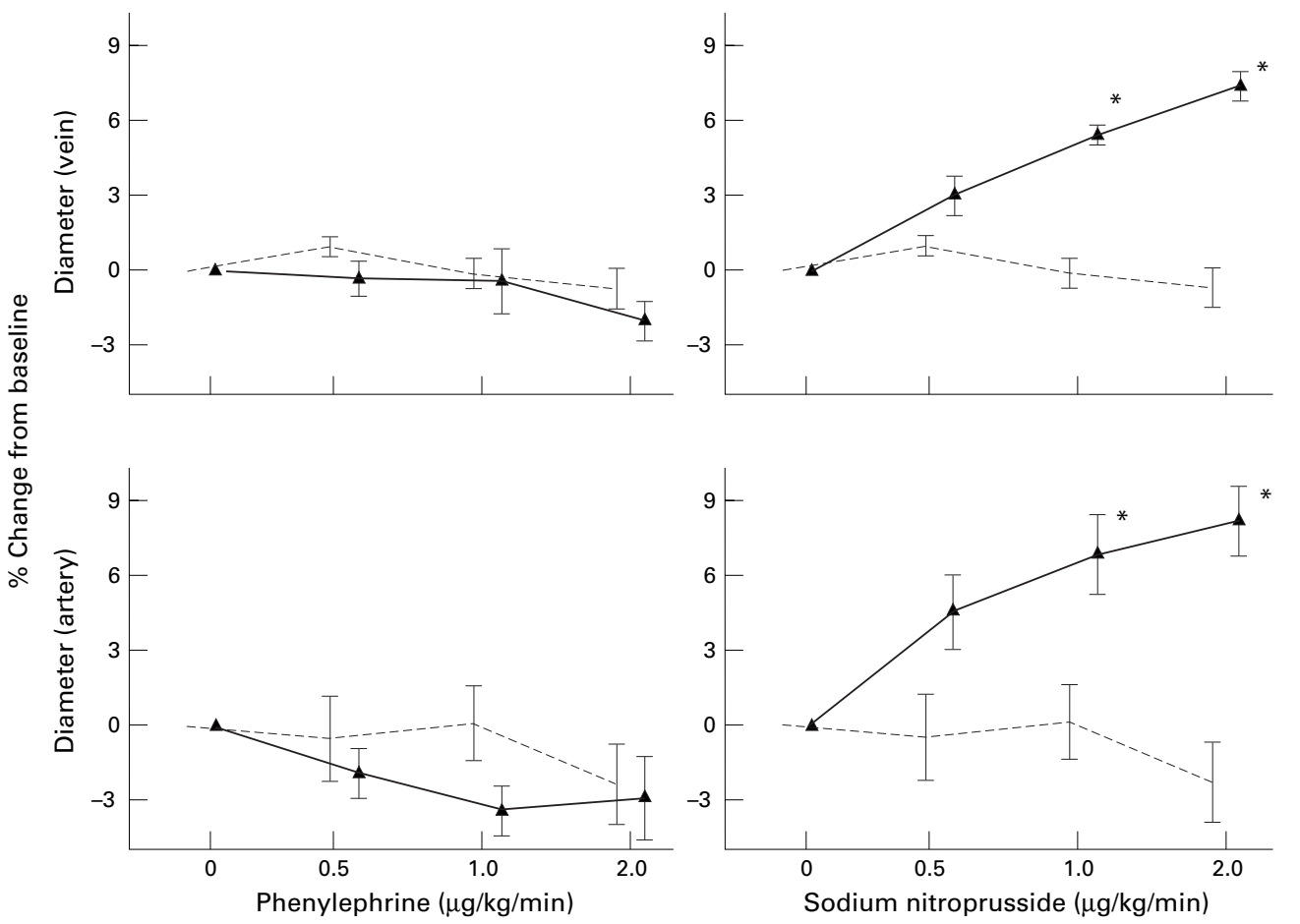

Figure 1 Effect of stepwise increasing doses of placebo (broken lines), phenylephrine, or sodium nitroprusside (solid lines) on retinal venous and arterial diameters. Data are presented as means $(S E M)(n=9)$. At the highest dose of phenylephrine and sodium nitroprusside only seven subjects were eligible for analysis. Asterisks indicate significant changes versus placebo.

$\kappa=(\mathrm{vs}-\mathrm{ve}) /(\mathrm{vs}+\mathrm{ve}+2 \times \mathrm{vm})$

The higher the intraclass correlation coefficients the better the reproducibility of the method. A $\kappa$ of 1 reflects perfect reproducibility. The intraclass correlation coefficient is a generally accepted measure of reliability and is considered more appropriate than older methods, such as $\chi^{2}$, percentage agreement, product moment correlation, or Yules's Y.$^{10}$ Short term variability ( $\mathrm{Ks}$ ) was calculated from the four measurements performed on the placebo study day. Day to day variability $(\kappa d)$ was calculated from the baseline measurements of the three study days. As an additional measure of variability, the coefficient of variation, was calculated for short term and day to day data. For this purpose individual coefficients of variation were calculated from the four readings at the placebo day and the three baseline readings of the three study days, respectively. The mean of these individual data are presented.

Effects of the haemodynamic model drugs were assessed by repeated measure ANOVA versus placebo and by repeated measure ANOVA versus baseline. Post hoc comparisons were done using paired $t$ tests and Bonferroni's correction for multiple comparisons. For data description drug induced changes were expressed as percentage change from baseline. Results are given as mean (SEM). p $<0.05$ was considered the level of significance.

\section{Results}

The intraclass correlation coefficients and the coefficients of variation for the measurement of retinal haemodynamic variables are presented in Table 1. Short term measurements of retinal vessel diameters with the Zeiss retinal vessel 

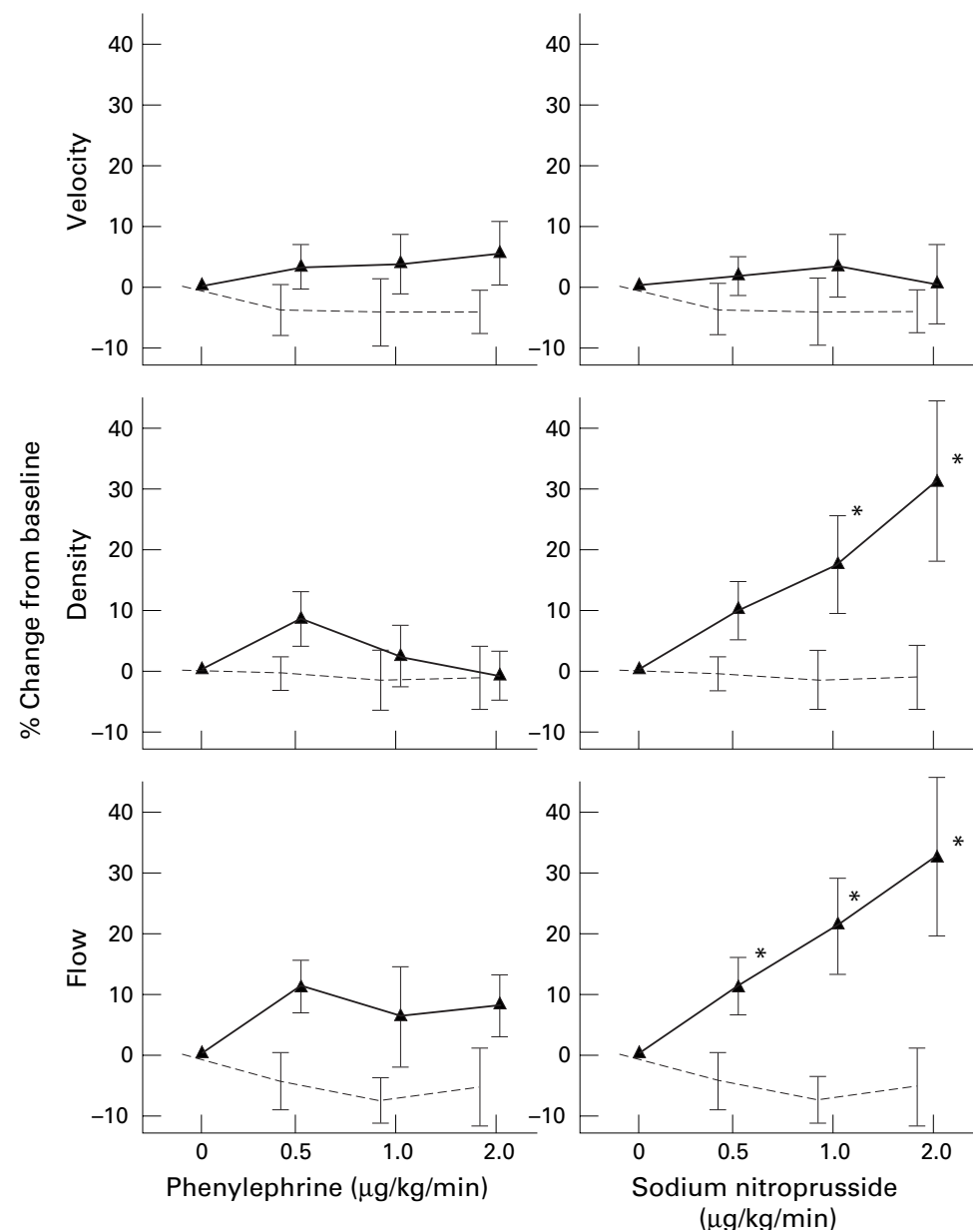

Figure 2 Effect of stepwise increasing doses of placebo (broken lines), phenylephrine, or sodium nitroprusside (solid lines) on leucocyte velocity, density of leucocytes, and leucocyte flow. Data are presented as means (SEM) $(n=9)$. At the highest dose of phenylephrine and sodium nitroprusside only seven subjects were eligible for analysis. Asterisks indicate significant changes versus placebo.

analyser are highly reproducible. The day to day variability of measurements obtained with the Zeiss retinal vessel analyser is considerably higher, but a $\kappa$ coefficient of more than 0.85 and a coefficient of variation in the order of $5 \%$ still indicates high reproducibility. As evidenced from all calculated variables results of diameter measurements in veins are more reproducible than in arteries. Short term reproducibility of measurements with the blue field entoptic technique is also acceptable, but it should be recognised that our study cohort represents a positive selection with regard to the ability of matching their own perception with the particles seen on the computer screen.

There were no significant differences between baseline ocular haemodynamic variables on the three study days (Table 2). Placebo had no consistent effect on ocular or systemic haemodynamics. In two subjects the phenylephrine infusion had to be discontinued at the highest dose owing to drug related side effects. In these subjects a pronounced decrease in pulse rate was observed and ocular haemodynamics could not be assessed. Hence, results presented for $2 \mu \mathrm{g} / \mathrm{kg} / \mathrm{min}$ phenylephrine are from seven subjects only. As expected phenylephrine significantly and dose dependently increased systolic $(\mathrm{p}<0.001)$ and diastolic blood pressure ( $p<0.001$, Table 3$)$. This rise in systemic blood pressure was paralleled by a pronounced decrease in pulse rate $(\mathrm{p}=0.002$, Table 3). By contrast, phenylephrine did not alter retinal venous diameter (Fig 1). However, a tendency towards a decrease in arterial diameter was observed during phenylephrine administration, but this effect did not reach the level of significance. Phenylephrine did not affect velocity, density, or flow of retinal leucocytes as assessed with the blue field entoptic technique. The drug, however, tended to increase retinal leucocyte flow, but the effect was less than $10 \%$ and not dose dependent.

In two subjects the infusions of sodium nitroprusside at the highest dose of $2 \mu \mathrm{g} / \mathrm{kg} / \mathrm{min}$ had to be discontinued owing to a pronounced systemic hypotensive response. Hence, only seven subjects were eligible for analysis at this dose. As expected, sodium nitroprusside induced a systemic hypotensive response with a more pronounced effect on diastolic $(\mathrm{p}<0.001$, Table 3) than on systolic blood pressure $(\mathrm{p}=0.013)$. A significant increase in pulse rate was also observed during drug administration $(\mathrm{p}<0.001$, Table 3). Sodium nitroprusside induced a dose dependent increase in both venous and arterial retinal diameter $(p<0.001$ each, Fig 1). The effect of the lowest dose of sodium nitroprusside on retinal venous diameter $(3.0 \%(0.8 \%))$ was significant versus baseline $(p=0.023)$, but not versus placebo. The effect of $1 \mu \mathrm{g} / \mathrm{kg} / \mathrm{min}$ sodium nitroprusside $(5.4 \%(0.4 \%))$ was highly significant versus baseline and placebo $(p<0.001$ each). The effect of the lowest dose of sodium nitroprusside on retinal arterial diameter $(4.5 \%(1.5 \%))$ was again significant versus baseline $(p=0.021)$, but not versus placebo. The effect of $1 \mu \mathrm{g} / \mathrm{kg} / \mathrm{min}$ sodium nitroprusside on retinal arterial diameter $(6.8 \%(1.6 \%))$ was significant versus baseline $(p=0.002)$ and placebo $(p=0.015)$. Sodium nitroprusside also induced a dose dependent increase in retinal leucocyte flow ( $p=0.024$, Fig 2 ). This effect was caused by an increase in retinal leucocyte density $(p=0.001)$, whereas retinal leucocyte velocity was virtually unchanged by the nitric oxide donor.

\section{Discussion}

The main purpose of the present study was to investigate the reproducibility and sensitivity of retinal vessel diameter measurements with the new Zeiss retinal vessel analyser. Our results indicate that the reproducibility in healthy subjects is higher for retinal veins than for retinal arteries. This could be a resolution size phenomenon, because in comparable fundus locations the veins are larger than the arteries. In addition, this may be related to the more pulsatile blood flow in the arterial tree, which leads to more pronounced vessel diameter changes during the cardiac cycle. ECG triggering $^{5}$ may therefore reduce short term variability of retinal vessel measurements with the Zeiss retinal vessel analyser. Nevertheless we obtained high short term reproducibility for vessel size determinations in both arteries and veins in the present trial. Day to day reproduc- 
ibility was considerably smaller, which might be related to difficulties in reproducing the relative position between the fundus camera and the subject's head.

The sensitivity of vessel size determinations with the Zeiss retinal vessel analyser was investigated using two model drugs in the present study. Our data indicate that in a double masked placebo controlled crossover trial in nine healthy subjects changes between 3\% and $5 \%$ in retinal venous diameters and changes between $5 \%$ and $7 \%$ in arterial diameters can be detected with this system. As retinal blood flow is dependent on the cross sectional area of the vessel our data indicate that changes in retinal blood flow in the order of $6-10 \%$ and $10-14 \%$ are detectable if measurements are taken from retinal veins and arteries, respectively. However, for a calculation of blood flow in a retinal vessel additional knowledge on blood speed is required. Combination of continuous laser Doppler velocimetry ${ }^{11}$ with the Zeiss retinal vessel analyser could, however, provide a technique for the real time assessment of blood flow in retinal vessels.

In comparison with previously realised methods for the assessment of retinal vessel diameters the Zeiss retinal vessel analyser provides several advantages. The continuous recording of vessel size allows quantification of effects of pharmacological or physiological interventions with high time resolution. In addition, vessel segments as well as different blood vessels can be investigated simultaneously. The Zeiss retinal vessel analyser does, however, not allow absolute vessel size measurements. This does not limit the applicability of the method for pharmacodynamic studies, but may limit its use in cross sectional studies. The clinical value of this instrument for monitoring eye diseases such as diabetic retinopathy, retinal artery and vein occlusion, or glaucoma remains, however, to be shown.

In the present study phenylephrine, an $\alpha$ receptor agonist, did not change retinal vessel diameters or retinal perifoveal leucocyte flow as evidenced from our measurements with the blue field technique. The lack of effect of phenylephrine on retinal haemodynamic variables could be for two reasons. There is evidence from several studies that retinal blood flow shows some autoregulation in response to an increase in perfusion pressure. ${ }^{12-14}$ Hence, the phenylephrine induced increase in arterial blood pressure may be counteracted by counterregulatory vasoconstriction. Additionally, there is evidence that adrenergic $\alpha$ receptors are present in retinal blood vessels ${ }^{15}$ and that phenylephrine causes contraction of bovine retinal resistance arteries in vitro. ${ }^{16}$ Hence, direct pharmacological effects of the $\alpha$ receptor agonist may also contribute to our results.

Sodium nitroprusside increased retinal venous and arterial diameters and retinal leucocyte flow, although it induced a potent systemic hypotensive response in the subjects under study. Counterregulatory vasodilatation could contribute to this effect, as retinal blood flow is autoregulated in response to a decrease in ocular perfusion pressure. ${ }^{17}$ Nevertheless, the present study indicates retinal vasodilatation in response to nitric oxide release. This finding is compatible with a variety of in vitro and in vivo studies indicating that nitric oxide is an important regulator of retinal ${ }^{18-25}$ and choroidal vascular tone. ${ }^{26-34}$ Interestingly, the effect of sodium nitroprusside on retinal leucocyte flow was paralleled by an increase in leucocyte density in the perifoveal area. This is compatible with our observation of vasodilatation at the level of the larger retinal vessels in response to the nitric oxide donor. Comparing the results obtained with these two techniques, one has to consider that an approximately $10 \%$ increase in vessel diameter indicates an approximately $20 \%$ increase in retinal blood flow through the major vessels, if blood velocity is assumed constant.

In conclusion we have shown that the Zeiss retinal vessel analyser provides a satisfactory reproducibility and sensitivity for pharmacodynamic studies. In addition, our data indicate that nitric oxide is a potent vasodilator in human retinal vessels.

The authors thank Zeiss Jena for the loan of the Zeiss RVA system. Particularly we thank Werner Infanger from Zeiss Vienna for his support. In addition, we thank Walter Vilser and Thomas Riemer for their extensive introduction to the operation of the RVA. Excellent technical assistance from Susan Entlicher, RN, and Ursula Graselli, RN, is acknowledged.

1 Delori FC, Fitch KA, Feke GT, et al. Evaluation of micrometric and densitometric methods for measuring the width of retinal vessel images on fundus photo
Graefes Arch Clin Exp Ophthalmol. 1998;226:393-9.

2 Chen HC, Patel V, Wiek J, et al. Vessel diameter changes during the cardiac cycle. Eye 1994;8:97-103.

3 Rassam SMB, Patel V, Brinchmann-Hansen O, et al. Accurate vessel width measurement from the fundus photographs: a new concept. Br f Ophthalmol 1994;78:24-

4 Suzuki Y. Direct measurement of retinal vessel diameter: comparison with microdensometric methods based on fundus photographs. Surv Ophthalmol 1995;39:S57-65.

5 Dumsky MJ, Aldington SJ, Dore CJ, et al. The accurate assessment of changes in retinal vessel diameter using multiple frame electrocardiograph synchronised fundus photography. Curr Eye Res 1996;15:625-32.

6 Formaz F, Riva CE, Geiser M. Diffuse luminance flicker Formaz F, Riva CE, Geiser M. Diffuse luminance flicker
increases retinal vessel diameter in humans. Curr Eye Res 1997; 16:1252-7.

7 Schmetterer L, Wolzt M, Salomon A, et al. Effect of isoproterenol, phenylephrine, and sodium nitroprusside on fundus pulsations in healthy volunteers. $\mathrm{Br} \mathcal{F}$ Ophthalmol 1996;80:217-23.

8 Vilser W, Riemer T, Bräuer-Burchardt C, et al. Retinal vessel analyzer (RVA) - a new measuring system for examination of local and temporal vessel behaviour. Invest Ophthalmol Vis Sci 1997;38:S1050.

9 Riva CE, Petrig BL. Retinal blood flow: laser Doppler velocimetry and blue field entoptic technique. Chapter 20 . In: Masters B, ed. Noninvasive diagnostic techniques in ophthalmology. New York: Springer, 1990.

10 Kramer MS, Feinstein AR. Clinical biostatistics. Clin Pharmacol Ther 1981;29:111-23.

11 Riva CE, Grunwald JE, Sinclair SH, et al. Fundus camera based LDV. Appl Opt 1981;20:117-20.

12 Riva CE, Grunwald JE, Petrig BL. Autoregulation of human retinal blood flow. An investigation with laser Doppler retinal blood flow. An investigation with laser Doppler
velocimetry. Invest Ophthalmol Vis Sci 1986;27:1706-12.

13 Rassam SMB, Patel V, Kohner EM. The effect of experimental hypertension on retinal vascular autoregulation in humans:a mechanism for the progression of diabetic retinopathy. Exp Physiol 1995;80:53-68.

14 Dumsky MJ, Eriksen JE, Dore CJ, et al. Autoregulation in the human retinal circulation: assessment using isometric exercise, laser Doppler velocimetry, and computer-assisted image analysis. Microvasc Res 1996;51:378-92.

15 Forster BA, Ferrari-Dileo G, Anderson DR. Adrenergic alpha ${ }_{1}$ and alpha $a_{2}$ binding sites are present in bovine retinal blood vessels. Invest Ophthalmol Vis Sci 1987;28:1741-6.

16 Hoste AM, Boels PJ, Brusaert DL, et al. Effect of alpha-1 and beta agonists on contraction of bovine resistance arteries in vivo. Invest Ophthalmol Vis Sci 1989;30:44-50.

17 Riva CE, Sinclair SH, Grunwald JE. Autoregulation of retinal circulation in response to decrease of perfusion pressure. Invest Ophthalmol Vis Sci 1981;21:34-8.

18 Granstam E, Wang L, Bill A. Vascular effects of endothelin-1 in the cat;modification by indomethacin and L-NAME. Acta Physiol Scand 1993;148:165-76. 
19 Seligsohn EE, Bill A. Effects of NG-nitro-L-arginine methyl ester on the cardiovascular system of the anaesthetized rabest releasing hormone. Br f Ophthalmol 1993;109:1219-25.

20 Haefliger IO, Zschauer A, Anderson DR. Relaxation of retinal pericyte contractile tone through the nitric oxide-cyclic guanosine monophosphate pathway. Invest Ophthalmol Vis Sci 1994;35:991-7.

21 Chakravarthy U, Stitt AW, McNally J, et al. Nitric oxide synthase activity and expression cells and pericytes. Curr Eye Res 1995;14:285-94.

22 Donati G, Pournaras CJ, Munoz JL, et al. Nitric oxide controls arteriolar tone in the retina of the miniature pig. Invest Ophthalmol Vis Sci 1995;36:2228-37.

23 Gidday JM, Zhu Y. Nitric oxide does not mediate autoregulation of retinal blood flow in newborn piglets. Am f Physio 1995;269:H1065-72.

24 Roufail E, Stringer M, Rees S. Nitric oxide synthase immunoreactivity and NADPH diaphorase staining are colocalised in neurons closely associated with vasculature in rat and human retina. Brain Res 1995;684:36-46.

25 Grunwald JE, DuPont J, Dreyer EB. Effect of chronic nitrate treatment on retinal vessel caliber in open angle nitrate treatment on retinal vessel caliber in
glaucoma. Am $\mathcal{f}$ Ophthalmol 1997;123:753-8.

26 Deussen A, Sonntag M, Vogel R. L-arginine derived nitric oxide: a major determinant of uveal blood flow. Exp Eye Res 1993;57:129-34
27 Mann RM, Riva CE, Stone RA, et al. Nitric oxide and choroidal blood flow regulation. Invest Ophthalmol Vis Sci 1995;36:925-30.

28 Zagvazdin YS, Fitzgerald ME, Sancesario G, et al. Neural nitric oxide mediates Edinger-Westphal nucleus evoked ncrease in choroidal blood flow in the pigeon. Invest Ophthalmol Vis Sci 1996;37:666-72.

29 O'Brien C, Kelly PAT, Ritchie IM. Effect of chronic inhibition of nitric oxide synthase on ocular blood flow and glu(t) 71 .

30 Schmetterer L, Krejcy K, Kastner J, et al. The effect of nitric oxide synthase inhibition on ocular fundus pulsations in man. Exp Eye Res 1997;64:305-12.

31 Schmetterer L, Findl O, Fasching P, et al. Nitric oxide and ocular blood flow in patients with IDDM. Diabetes 1997;46:653-8.

32 Schmetterer L, Krejcy K, Findl O, et al. Role of NO in the $\mathrm{O}_{2}$ and $\mathrm{CO}_{2}$ responsiveness of cerebral and ocular circulation in humans. Am f Physiol 1997;273:R2005-112.

33 Kiel JW, Maldonado AL. Hexamethonium, nitric oxide and choroidal autoregulation. Invest Ophthalmol Vis Sci 1998; 39:S1121

34 Koss MC. Role of nitric oxide in maintenance of basal anterior choroidal blood flow in rats. Invest Ophthalmol Vis Sci 1998;39:559-64. 